resection of the epileptogenic tubers and epileptogenic foci is successful in the control of seizures in $78 \%$ (range $43-100 \%$ ), and results in reduction of seizure frequency in $20 \%$ (10$45 \%$ ). Quality of life is improved in $95 \%$ as a result of seizure control or reduction in medication. Accurate presurgical testing is essential and involves surface videoelectroencephalogram, MRI, positron emission tomography, single-photon emission computed tomography, magnetoencephalography, and invasive EEG monitoring. Early detection and resection of medically refractory epileptogenic tubers can prevent deterioration of cognitive functioning, improve behavior and socialization, and offer a better qualiy of life. (Romanelli P, Verdecchia M, Rodas R, et al. Epilepsy surgery for tuberous sclerosis. Pediatr Neurol 2004;31:239-247). (Respond: Dr Paolo Curatolo, Pediatric Neurology, University of Rome "Tor Vergata,"V le Oxford 81, 00133, Rome, Italy).

COMMENT. Early effective seizure control may significantly reduce the adverse effects on cognition and development associated with refractory recurrent seizures in tuberous sclerosis. With new presurgical investigative techniques, it is now possible to select patients who may be amenable to resection of multiple epileptogenic tubers. In patients not suitable for resection, anterior callosotomy, vagal nerve stimulation, and radiosurgery are alternative methods suggested.

\title{
RISK-BENEFITS OF OXCARBAZEPINE VS CARBAMAZEPINE
}

The clinical differences between oxcarbazepine (OXC) and carbamazepine (CBZ) are reviewed in terms of efficacy, tolerability, and interaction with other drugs in a study at Universitat Bonn, Berlin, Germany. OXC (Trileptal) is used as monotherapy or adjunctive therapy for the treatment of partial seizures with or without secondary generalization in adults and children above 4 years (USA) or 6 years (Europe) of age. The mechanism of action of $\mathrm{OXC}$ resembles CBZ in involving sodium channel blockade; it differs in the type of calcium channel modulation. OXC metabolizes by reduction to the monohydroxy derivative (MHD) and is excreted in the urine after conjugation as a glucuronide, with no autoinduction. $\mathrm{CBZ}$ is oxidized to 10,11 -epoxide and is hydrolyzed in the liver. Hepatic metabolism of $\mathrm{OXC}$ and enzyme induction are minimal, so that drug combinations show little interaction. $\mathrm{OXC}$ is better tolerated and its risk-benefits are superior to CBZ. It causes fewer skin rashes than $\mathrm{CBZ}$; rashes occurred in $3 \%$ of patients taking OXC compared to $7 \%$ of those treated with CBZ. Cross-reactions may occur, and in patients with known hypersensitivity to CBZ, therapy with OXC should be avoided. The efficacy of oral contraceptives may be impaired by $\mathrm{OXC}$, and risks of teratogenicity remain to be determined. Hyponatremia $(<135 \mathrm{mEq} / \mathrm{L})$ has occurred more frequently with OXC (30/104 patients, $29 \%)$ than with CBZ $(69 / 479$ patients, $14.4 \%)$. Clinically significant hyponatremia $(<125 \mathrm{mmol} / \mathrm{L})$ occurred in $2.7 \%$ of adults with previously normal values; it is less common in children $(0.2 \%)$. (Schmidt D, Elger CE. What is the evidence that oxcarbazepine and carbamazepine are distinctly different antiepileptic drugs? Epilepsy \& Behav 2004;5:627-635). (Respond: E-mail: dbschmidt@tonline.de).

COMMENT. The authors recommend OXC as preferable to $\mathrm{CBZ}$ because of proven efficacy and lower incidence of side effects. In a multicenter, randomized, double-blind, parallel group trial of 62 newly diagnosed children ages 5 to 17, OXC (Trileptal) in a mean 
dose of $20 \mathrm{mg} / \mathrm{kg} /$ day (range, $8-39 \mathrm{mg} / \mathrm{kg} /$ day) provided a $>75 \%$ reduction in partial seizures in $84 \%$ patients, and $100 \%$ reduction in $60 \%$. (Report of responses from neurologists provided by Bergman S, Novartis). As add-on therapy in 267 children with partial seizures, a $>75 \%$ reduction in seizure frequency occurred in $27 \%$ vs $7 \%$ in patients receiving placebo. Sixty-one percent of neurologists were already using Trileptal as monotherapy for partial seizures in children ages $4-16$, and $68 \%$ use it as adjunctive therapy. In a review of monotherapy in epilepsy and the role of newer antiepileptic drugs, OXC is as effective as conventional AEDs at controlling partial seizures and is better tolerated (Vasquez B. Arch Neurol Sept 2004;61:1361-1365).

Oxcarbazepine exacerbates myoclonic and absence seizures in juvenile idiopathic generalized epilepsies (IGE), in a report from Montpellier and Marseille, France (Gelisse P et al. Epilepsia 2004;45:1282-1286). Six patients, ages 6 to 19 years, with juvenile IGE were affected; 5 had worsening of myoclonic jerks, 3 had exacerbation of absence seizures (one had absence status); and 3 had a slight increase in generalized tonic clonic seizures.

\section{RISKS OF ACADEMIC UNDERACHIEVEMENT IN EPILEPSY}

The relation between neuropsychological functioning and academic achievement and the role of family environment, seizures, and psychosocial variables were examined in 173 children with chronic epilepsy, ages 8 to 15 years, at Purdue School of Science; Indiana University School of Medicine, Indianapolis; and University of Wisconsin-Madison School of Medicine, WI. Verbal/Memory/Executive function and Rapid Naming/Working Memory were strongly correlated with reading, math, and writing ability, whereas Psychomotor function predicted writing ability only. Family environment affected the impact of neuropsychological deficits (NPD) on writing and reading; NPD had a lesser impact on

achievement of children from supportive/organized homes compared to unsupportive/disorganized homes. (Fastenau PS, Shen J, Dunn DW, et al. Neuropsychological predictors of academic underachievement in pediatric epilepsy: moderating roles of demographic, seizure, and psychosocial variables. Epilepsia 2004;45:1261-1272). (Reprints: Dr PS Fastenau, Psychology (LD 124), Indiana University Purdue University Indianapolis, 402 N Blackford St, Indianapolis, IN 46202).

COMMENT. Children with epilepsy who have neuropsychological deficits and live in disorganized unsupportive home environments are particularly at risk for academic underachievement. Aggressive family intervention and counseling to provide more supportive environments might result in improved academic outcome. Involving the family in the learning process is known to benefit learning, and a more organized family life can promote better sleeping habits and full compliance with medication schedules.

Changes in left temporal interictal epileptiform activity during and after cognitive tasks are reported in an 18-year-old male with intractable complex partial seizures (Boniface SJ, et al. J Neurol Neurosurg Psychiatry 1994;57:227-228). (See Ped Neur Briefs April 1994). Activation or suppression of EEG focal spike discharges in relation to psychological testing may have a role in treatment of children with reading and other learning disabilities, and in presurgical assessment of patients with intractable epilepsy. 